\title{
Editorial
}

\section{Molecular Imaging In Vivo}

In vivo imaging such as positron emission tomography (PET) and MRI, has been playing an important role in diagnosing diseases and monitoring treatment for decades. Recently, in vivo imaging is widening its field through revealing molecular event in cells and tissues. Molecular imaging is used for earlier detection, characterization of disease and an earlier assessment of treatment efficacy through imaging molecular/cellular events in living organisms.

Molecular imaging is useful not only for clinical studies but also for developing new drugs and new treatment modalities such as gene or stem cell therapy. Pre-clinical molecular imaging using animals such as mice shows biodistribution, pharmacokinetics, mechanism of action, and efficacy in vivo. Nanoparticles are able to carry fluorescent dye, radioisotope, drugs, genes, and targeting biomarkers on their surface and inside. Since each modality such as PET, MRI, and optical imaging has its own limitation, not a single modality covers all the scopes. Multimodal in vivo imaging using different modalities at once will be a solution. If we combine nanotechnology with imaging technology, personalized targeting therapy can be guided by multimodal multiplexing in vivo imaging.

Molecular imaging should have been based on multimodal imaging at free will of investigators in the field, however, this versatility waited for the advent of a new era of nanotechnology. Recent advances of nanotechnology and its application to the biotechnology enabled us to use combination of various modalities to suit the purposes of this investigation of molecular imaging. Sometimes it tracks implanted stem cells, in other times traces their fates in vivo, or reveals the changes of microenvironment such as angiogenesis. This combined technology still gives us a hope and ambition to visualize in vivo cellular processes in detail with excellent spatial resolution and also on the fine temporal axis. At first they will focus their attention to visualize the fates of implanted cells or even tissues (or cell-scaffolds) but finally they will be sure to get successful in visualizing the fates of endogenous cells and tissues.

Traditionally, nuclear medicine successfully depicted the behavior of organs using radioisotope-labeled small molecule, however, recently using multimodal nanoparticles, which are sometimes combined with small molecules, we could visualize intracellular molecular processes. How to make this technology visualize multiple intracellular molecular processes at the same time is the next challenge ahead in the field. We call it multiplexing of molecular imaging in vivo. Based on freedom used for multimodal molecular imaging in vivo, multiplexed imaging will open a huge vault of so-far-unknown hidden sanctuary of information associated with cellular molecular processes.

In this Hot Topic issue, I have collected various subjects to make a series of reviews and recruited the willing young investigators who dared to take efforts to summarize the progresses of this field. While there are rare real progresses from the top-researchers in the field, even the best progresses are confined to the stages of proof-of-concept and often leave lots of unanswered questions behind. The 'normal' investigators like myself who are very much interested in the clinical application of this technology in men feel astray while following the direction of those pioneers. This might be the reason of the make-up of this kind of series of summary regarding this field's progress.

I would like to ask the readers to understand that the scopes of each review article are sometimes overlapped, this was not the fault of each reviewer, but represented the situation where this field of 'molecular imaging in vivo' is. Just enjoy the different perspectives of each reviewer and combine the hasty apparent impressions from each perspective on your own to get a glimpse of new future direction of investigation in this field. The reviews should not be limited to the field of 'cancer research' but were meant to include the progresses of brain, heart and regenerative medicine, or tissue engineering and also the field of chronic degenerative diseases of every bodily organ.

At first, I and my colleague managed to deal with the subject of 'Multimodal molecular imaging in vivo' in a general viewpoint. This was an introduction for the following articles but still focused on the multimodality in its broad scopes. The second topic of 'PET/MRI/Fluorescent molecular imaging' followed with the intention of clear and brief description of representative PETMRI-fluorescence technology which will possibly be used for new PET/MRI machines.

The contribution entitled 'Molecular imaging using bioluminescence' explains the topic of theragnostics using bioluminescence technology for molecular imaging. This was written by an expert who recently established usage of 'bactovector' technology in the field. The next, 'Angiogenesis imaging' should combine the expertise of imaging and chemistry and was summarized by a combined effort of a young nuclear physician and an established expert in the field of radiochemistry. This subject represents the success of the investigators in the field of nuclear/ molecular imaging to find the right biomarker and imaging technology especially using small molecules to visualize molecular processes at the surface of cells. The next subject of 'Stem cell imaging' reviewed by one of the best Chinese investigators of the present time summarized the recent progresses in stem cell tracking. Though we wish the technology should go ahead of the time, it used to accompany the progresses of stem cell research, and the field of stem cell research had yet to catch up with the progresses in tissue engineering, regenerative medicine and organomics or much more in molecular biology. The article on 'Molecular imaging using PET/MRI particles' recapitulated 
the necessity of using nanoparticles in our field for tracking cells, tracing molecular processes during differentiation or proliferation and also multiple molecular biological processes. This review contains a short summary of a few recent investigations and various future perspectives of 'to-do-next' items. For the translation of all the above imaging stuffs in vivo to human or clinical situations, we needed to invent 'New hybrid cameras for multimodal imaging' and this aspect was excellently reviewed by a rising young expert in the field. I am sure the readers will like it, whether they are physicians or physicists. Standard and classical review of 'Radiopharmaceuticals for molecular imaging' was added to enhance the understanding of pharmaceutical issues as on easy-to-understand introduction by another young radiochemist.

In summary, I am quite sure we just opened a new opportunity of understanding of this new field and will have a chance to see a new possibility. But we have more things to do than we did in this emerging newly-delivered subject of molecular imaging in vivo as it is in its infancy. I wish I could summarize further progress dedicated to each subfield later, organ-typically or technologically in the near future.

\section{Dong Soo Lee}

(Guest Editor)

Department of Molecular Medicine and Biopharmaceutical Sciences Seoul National University

Seoul, 110-744

Republic of Korea

E-mail: dsl@plaza.snu.ac.kr

(C) Dong Soo Lee; Licensee Bentham Open.

This is an open access article licensed under the terms of the Creative Commons Attribution Non-Commercial License (http: //creativecommons.org/licenses/by-nc/ 3.0/) which permits unrestricted, non-commercial use, distribution and reproduction in any medium, provided the work is properly cited. 